Man and Nature

L'homme et la nature

\title{
L'Étrange don du Marquis de Sade à la Révolution et à l'Empire : son théâtre
}

\section{Marie-France Silver}

Volume 6, 1987

URI : https://id.erudit.org/iderudit/1011880ar

DOI : https://doi.org/10.7202/1011880ar

Aller au sommaire du numéro

Éditeur(s)

Canadian Society for Eighteenth-Century Studies / Société canadienne d'étude du dix-huitième siècle

ISSN

0824-3298 (imprimé)

1927-8810 (numérique)

Découvrir la revue

Citer cet article

Silver, M.-F. (1987). L'Étrange don du Marquis de Sade à la Révolution et à l'Empire : son théâtre. Man and Nature / L'homme et la nature, 6, 209-223. https://doi.org/10.7202/1011880ar

Copyright (C Canadian Society for Eighteenth-Century Studies / Sociéte canadienne d'étude du dix-huitième siècle, 1987
Ce document est protégé par la loi sur le droit d'auteur. L'utilisation des services d'Érudit (y compris la reproduction) est assujettie à sa politique d'utilisation que vous pouvez consulter en ligne.

https://apropos.erudit.org/fr/usagers/politique-dutilisation/ 


\section{L'ÉTRANGE DON DU MARQUIS DE SADE À LA RÉVOLUTION ET À L'EMPIRE: SON THÉÂTRE}

En octobre 1799, s'adressant à Goupilleau de Montaigu, membre de la Convention, le citoyen Sade, homme de Lettres, met son théâtre au service de la patrie:

“On dit que ma plume a quelque énergie ... j'offre donc mes moyens à la Révolution, et les lui offre du meilleur de mon coeur."1

Si Sade était à cette date convaincu de son génie théâtral comme en témoigne sa correspondance, ${ }^{2}$ il n'avait rencontré comme dramaturge qu'un succès d'estime.

Quatre de ses pièces semblent avoir été acceptées par des théâtres publics ${ }^{3}$ et deux furent certainement représentées: il s'agit du drame Le Comte Oxtiern ou les dangers du libertinage qui fut joué en 1791 au théâtre Molière de la Porte Saint-Martin et qui fut repris en 1799 au thétre de Versailles et du Suborneur qui fut représenté en 1792 au Théâtre Italien.

La correspondance de Sade révèle qu'à partir de 1799, faire jouer ses pièces, devient pour l'auteur une véritable obsession.

Harcelant directeurs de théâtre et comédiens, s'impatientant de leurs critiques, c'est cette année-là qu'il eut recours à des hommes politiques pour qu'ils fassent pression sur la Comédie Française pour l'obliger à produire sa tragédie Jeanne Laisné. 
"Il faut que le gouvernement la fasse jouer d'autorité comme pièce patriotique", réclame Sade. ${ }^{4}$ Les raisons de cet empressement à se faire jouer nous aident à mieux comprendre cette période difficile de la vie de l'auteur.

Mis par erreur en 1797 sur la liste des immigrés du Vaucluse, il voit ses biens confisqués. A partir de ce moment il doit vivre d'expédients: pour 40 sous par jour il travaille comme souffleur au théâtre de Versailles. Quand il perd cet emploi il est obligé d'entrer à l'hôpital de cette ville pour éviter de mourir de faim. Pour retrouver ses biens, le cidevant marquis doit prouver son civisme et son adhésion aux valeurs révolutionnaires. Ce n'est pas la première fois que Sade se trouve dans cette situation: emprisonné sous Robespierre pour modérantisme et condamné à mort, il avait retrouvé la liberté en 1794 grâce au certificat de civisme fourni par la section révolutionnaire de la place Vendôme à laquelle il s'était joint en 1790 et dont il avait été successivement secrétaire puis président. Mais en 1797 la situation est beaucoup plus délicate.

Cette année-là en effet La Nouvelle Justine et L'Histoire de Juliette paraissent en Hollande. Le scandale est immédiat et les éditions clandestines s'arrachent à Paris. Dans cette période déjà difficile, Sade va devoir en plus, se défendre de la dangereuse accusation d'immoralité. Et c'est son théâtre qu'il va offrir à l'opinion publique pour se justifier. Le rapprochement de certaines dates est révélateur. En avril 1798 le Journal de Paris attribue à Sade la paternité de La Nouvelle Justine et en dénonce l'obscénité. Sade nie en être l'auteur ${ }^{5}$ et le 21 juillet 1798 il revendique dans ce même journal l'honneur d'avoir découvert le vrai nom de Jeanne Hachette, c'est-à-dire Jeanne Laisné, en faisant des recherches pour cette pièce dans les archives de Beauvais. ${ }^{6}$ En août et octobre 1799 les accusations de la presse se font extrêmement violentes pour dénoncer l'immoralité de La Nouvelle Justine et de son auteur. ${ }^{7}$ La presse se fait même l'écho de rumeurs qui veulent que Sade ait organisé un club de débauchés. Ainsi, confondant délibérément l'auteur avec les personnages de ses romans, forgeant à loisir des anecdotes odieuses pour mieux frapper l'opinion, la presse contribue à la formation de la légende du Sade monstrueux que les siècles suivants nous ont transmise. "Le tribunal d'Apollon" en appelle à la police pour arrêter les ventes clandestines de ces ouvrages et faire arrêter Sade. ${ }^{8}$ De 1798 à 1801 ce genre d'accusations se multiplie dans la presse. La tactique de Sade est d'essayer $\mathrm{d}^{\prime}$ intéresser l'opinion publique à son théâtre. Mais c'est en vain que Sade dramaturge va essayer de lutter contre sa propre légende. Les travaux de Jean Tulard ${ }^{9}$ montrent que c'est dans la période qui précéda l'établissement d'une direction générale de la librairie en 1810, 
que la France connut la censure la plus sévère de son histoire, puisque cette censure relevait uniquement de la police et n'était limitée par aucun règlement. La police exerçait donc seule et sans contrôle la surveillance de l'imprimerie et de la librairie. Au contrôle des livres politiques, libelles ou pamphlets, le préfet de police ajoutait celui "des ouvrages contraires aux bonnes moeurs".

Ce pouvoir absolu aide à mieux comprendre les circonstances de l'arrestation de Sade chez son libraire le 6 mars 1801 comme "auteur de l'infâme roman Justine et de l'ouvrage plus affreux encore Juliette". ${ }^{10}$

Interné à Sainte-Pélagie puis à Bicêtre il est finalement transféré en avril 1803 à l'asile psychiatrique de Charenton pour "démence libertine". Il venait grossir les rangs de cette catégorie de "fous pour raison $\mathrm{d}^{\prime}$ 'Etat"11, catégorie qui, à Charenton ne cesse d'augmenter jusqu'en 1806, année où les prisonniers politiques sont alors plus nombreux que les aliénés véritables. ${ }^{12}$

Continuant à voir dans son théâtre une source possible de réhabilitation sociale et politique, il persévère dans l'espoir de se faire jouer et pour mieux amadouer Napoléon, il retouche même certaines de ses pièces pour y incorporer les éloges les plus nauséabonds de l'Empereur et du Roi de Rome qu'il m'ait été donné de lire. ${ }^{13}$ Sans résultat d'ailleurs. Malgré les demandes réitérées de Sade, la commission sénatoriale de 1804 destinée à préserver la liberté de pensée et la liberté individuelle ne statue pas sur son cas. ${ }^{14}$ Et Napoléon qui avait en horreur l'auteur de Justine confirme par deux lettres signées de sa main en 1810 et 1812 la détention de Sade. ${ }^{15}$

Dans l'attitude de Sade envers son théâtre un des aspects les plus intéressants, me semble-t-il, est que l'auteur considérait son théâtre comme une source de salut personnel.

De la Bastille il écrivait en 1786:

"ce serait pour moi un grand plaisir sans doute de voir jouer mes ouvrages à Paris, et si je parvenais à y réussir, la réputation d'esprit que je me procurerais ferait peut-être oublier les travers de ma jeunesse et me réhabiliterait dans un sens. Cela m'occuperait beaucoup et me retirerait absolument de tout le reste. J'ose même dire que c'est le seul moyen, et la raison en est physique: il faut une force supérieure pour combattre une force puissante". ${ }^{16}$

Ce qui n'était alors qu'intuition va devenir conviction quand Sade, interné à Charenton, trouva dans le directeur, monsieur de Coulmiers, un protecteur qui partageait sa conception du théâtre comme thérapie possible de l'aliénation. Il semble qu'il y ait peut-être eu des spectacles organisés à l'asile par M. de Coulmiers avant l'arrivée de notre auteur. ${ }^{17}$ 
Mais le directeur fut ravi de trouver dans Sade un homme de théâtre qui avait fait jouer des pièces pendant la Révolution. L'enquête ordonnée par le ministère de l'Intérieur en 1808 sur le marquis de Sade met en évidence l'encouragement que M. de Coulmiers apportait à Sade et à son théatre:

M. de Coulmiers (écrit le lieutenant de police), dit même à cet égard qu'il a beaucoup d'obligation à Sade, parce que regardant la comédie comme un moyen curatif de l'aliénation d'esprit, il se trouve heureux d'avoir dans son hospice un homme capable de former à la scène les aliénés qu'il veut guérir par ce genre de remède. ${ }^{18}$

Les lettres échangées par les autorités de l'asile, le préfet de police et le ministre de l'Intérieur, attestent du rôle de Sade dans l'organisation de ces spectacles.

Ainsi Royer-Collard, médecin-chef, le 2 août 1808 au ministre de l'Intérieur:

Monsieur de Sade est le directeur de ce théâtre. C'est lui qui indique les pièces, distribue les rôles et préside aux répétitions. Il est le maître de déclamation des acteurs et des actrices et les forme au grand art de la scène. Le jour des représentations publiques, il a toujours un certain nombre de billets à sa disposition, il fait en partie les honneurs de la salle, il est même auteur dans les grandes occasions. ${ }^{19}$

Le rapport adressé par le préfet de police au ministre de l'Intérieur en septembre 1808, confirme ces activités. Les documents indiquent que la majorité des pièces appartenaient au répertoire traditionnel et que les invités venaient nombreux de Paris, attirés par la curiosité d'un tel spectacle, comme par la légende noire du marquis. ${ }^{20}$ Mais l'attitude libérale de $M$. de Coulmiers envers Sade, la croyance qu'il partageait avec cet auteur dans la valeur thérapeutique du théâtre, ne sont pas caractéristiques de tous les dirigeants de Charenton. Autour de RoyerCollard, qui se joint au personnel en 1808, va se former une forte opposition aux spectacles de l'asile et au rôle joué par Sade dans leur organisation. Sade, dans $L a$ Fête de l'amitié, la seule pièce écrite à Charenton pour les aliénés qui soit parvenue jusqu'à nous, prend la défense de $M$. de Coulmiers et affirme la valeur thérapeutique des spectacles de Charenton. Dans cette pièce étrange Sade met en scène Momus, le dieu de la folie, et les habitants de ses asiles et il fait à travers le personnage D'Orphanie un vibrant plaidoyer en faveur de ces spectacles. ${ }^{21}$ Mais le mouvement d'opposition à cette thérapie théâtrale est 
si actif auprès des autorités, qu'après de nombreuses enquêtes un arrêté ministériel du 6 mai 1813 interdit désormais tout spectacle dans la maison de santé de Charenton. ${ }^{22}$ Et le refus final que fait la Comédie Française l'année suivante de représenter Jeanne Laisné, ôte à Sade tout espoir d'effacer par son théâtre sa réputation de romancier.

Le côté moralisateur du théâtre sadien, qui exaspère les admirateurs de ses romans, est parfaitement compréhensible si on le replace dans le contexte de son époque. Il est aussi loin d'être sans intérêt pour l'histoire littéraire.

Dans la période où Sade essaie de se faire jouer il y a plus de trente ans que le néo-classicisme impose aux différentes formes artistiques, son optique moralisatrice.

A un mouvement déjà solidement implanté, la Révolution apporte son appui et consacre l'artiste dans son rôle d'éducateur. David n'avait-il pas déclaré à la Convention en 1793:

Le vrai patriote doit saisir avec avidité tous les moyens d'éclairer ses concitoyens et de présenter sans cesse à leurs yeux les traits sublimes de l'héroisme et de la vertu. ${ }^{23}$

La correspondance que Sade échange avec les directeurs de certains théâtres parisiens atteste de ces exigences. La pièce Le Boudoir se voit refuser par le théâtre de la rue Feydeau parce que "les caractères produiraient des impressions faites pour alarmer les amis des bonnes moeurs" ${ }^{24}$ La comédienne Julie Candeille partage cet avis:

"La petite pièce intitulée Le Boudoir est écrite agréablement mais je doute que le sujet, libre en lui-même, puisse être sans danger développé à la scène. On sait que les gens rassemblés sont d'autant plus sévères sur les moeurs qu'on leur représente qu'ils le sont moins dans le particulier". ${ }^{25}$

Le théâtre du Palais-Royal refuse Le Prévaricateur parce que le personnage de Philoquet est pardonné au dénouement:

Un tel homme ... n'est point susceptible d'indulgence, et doit être puni ... Ce pardon d'ailleurs, le public ne le partagerait pas. Il est juste et vertueux lorsqu'il est rassemblé, il veut que le crime subisse sa peine. Absoudre un Philoquet ce sera le révolter, détruire le but moral de votre pièce et donner un exemple dangereux. ${ }^{26}$

Cette exigence du public n'avait pas échappé à Sade qui explique ainsi 
et non sans humour, la ruine de Fonrose dans le dénouement du Capricieux:

L'on se serait infailliblement déchaîné contre nous si nous n'eussions pas écrasé notre marquis de Fonrose de toutes les foudres qui se trouvent sur sa tête. ${ }^{27}$

Le Misanthrope par amour, drame qui valut à Sade cinq ans d'entrées gratuites à la Comédie Française, n'y fut jamais représenté parce que le sujet en avait été trouvé trop "osé". "L'administration du Théâtre Français, écrit le directeur, ne peut se permettre de faire représenter aucun ouvrage douteux" ${ }^{28}$

Aux critères moraux, la période révolutionnaire ajoute des critères politiques dans l'évaluation d'une oeuvre: "tout ce qui rappelle le passé frappe désagréablement le public", écrit à Sade en 1791 le directeur du théâtre du Marais pour expliquer son refus du Prévaricateur. ${ }^{29} \mathrm{Il}$ n'est guère étonnant non plus que cette période exige des théâtres qu'ils exaltent le patriotisme. Le Moniteur du 11 novembre 1791 loue le théâtre Molière de son patriotisme: "ce théâtre depuis son ouverture, s'est distingué par le patriotisme et l'amour de la Révolution". ${ }^{30} \mathrm{C}^{\prime}$ est dans cet esprit que ce théâtre accepte de représenter Oxtiern. En 1814, dans son compte-rendu éliminatoire de Jeanne Laisné, la Comédie Française loue Sade d'avoir choisi un sujet patriotique mais lui reproche essentiellement d'avoir fait du maire de Beauvais un traître. ${ }^{31}$ La France ne pouvait admettre de traitre, même en littérature. Le 5 mars 1792 la représentation du Suborneur au Théâtre Italien se voit interrompre par les sans-culottes:

La faction jacobine a fait tomber le mois passé une pièce de moi au Théâtre Italien seulement parce qu'elle était d'un ci-devant. Ils parurent en bonnet de laine rouge. ${ }^{32}$

Le compte-rendu de cette manifestation dans le Journal des théatres du 10 mars confirme l'incident:

Pendant l'entracte nous avons vu des patriotes se coiffer d'un bonnet rouge ... Un de ceux qui en étaient coiffés a dit tout haut que ce bonnet rouge serait désormais dans les endroits publics le signe auquel se rallieraient les patriotes et surtout dans les spectacles, où l'aristocratie serait sans cesse combattue par les amis de la liberté. ${ }^{33}$

Héritier des Lumières, le théâtre de Sade aurait dû plaire aux révolu- 
tionnaires: attaques contre l'Ancien Régime; contre la cour; contre la corruption de la noblesse; ${ }^{34}$ contre l'arbitraire de la Justice; ${ }^{35}$ éloge du peuple; identification du plébéien à la vertu; ${ }^{36}$ autant de leitmotive qui se répètent de pièce en pièce. Et pour entretenir l'ardeur révolutionnaire, Sade avait même consacré trois de ses pièces au thème du patriotisme: deux tragédies Jeanne Laisné et Euphémie de Melun ou le siège d'Alger et une pièce lyrique Tancrède.

Mais le théâtre du marquis de Sade reflète aussi le goût du dixhuitième siècle finissant pour une certaine forme de sensibilité. Contemporain du roman noir anglais qu'il connaissait bien, ${ }^{37}$ son théâtre regorge de cachots perdus au fond de châteaux irlandais, ${ }^{38}$ de taudis londoniens, ${ }^{39}$ de forêts séculaires ${ }^{40}{ }^{4}$ 'auberges suédoises,${ }^{41}$ de bombardements, ${ }^{42}$ de pressentiments, ${ }^{43}$ de revenants, ${ }^{44}$ d'empoisonnements ${ }^{45}$ et de suicides. ${ }^{46}$ La poésie des tombeaux, chère au romantisme anglais, n'en est même pas absente et dans Henriette et Saint-Clair, on peut voir les amants sur le point de se suicider tracer leur épitaphe. Toute cette thématique du roman noir se retrouve également dans ses contes: Les Crimes de l'amour. ${ }^{47}$ Mais comme dans cet ouvrage, ce que Sade met en scène dans son théâtre, c'est avant tout le tragique de la passion. S'il y a relativement peu de violence physique sur la scène, c'est que la véritable violence s'exerce dans la torture des âmes rongées par la passion. Au romantisme du décor, de la mise en scène, des situations correspond en effet le romantisme des sentiments: passion tragique, passion folle, omniprésence de la passion dans ce théâtre. Lincée devient fou parce qu'il est séparé d'Orphanie, ${ }^{48}$ Fanni se suicide parce qu'elle a perdu son amant, ${ }^{49}$ Sophie et Desfrancs, Henriette et Saint-Clair voient planer sur eux le tabou de l'inceste, la passion de Derval le conduit au pied de l'échafaud. ${ }^{50}$ Face à l'injustice, aux préjugés et aux fausses valeurs de la société; face à l'hostilité de familles éprises de stériles traditions, les héros sensibles du théâtre sadien cherchent dans leur passion la justification de leur existence, la cohérence que le monde ne leur offre pas.

"Qu'eût été la vie sans lui?" s'intérroge Cécile, ${ }^{51}$ Derval parlant de son amour: "C'est pour lui que j'entreprends tout". ${ }^{52}$ "Que ferai-je au monde quand je ne $t^{\prime} y$ possèderai plus?" ${ }^{\prime 23}$ demande Saint-Clair à Henriette et, à son père pour lui faire accepter son amour pour la jeune fille:

Vous ne voudrez pas le malheur de votre fils et quand vous serez convaincu de toute la violence de sa passion ... quand vous serez persuadé que son amour et sa vie n'ont maintenant qu'un même principe et que vous le priverez du jour plutôt que d'étreindre la violence du feu qui le maîtrise ... vous vous ressouviendrez que vous êtes père. ${ }^{54}$ 
Comme chez Rousseau, la sensibilité, la passion valorisent les personnages. Dans cette optique, passion et vertu se confondent. "Mon amour est le plus pur encens que je crois offrir à la vertu", s'exclame Henriette. ${ }^{55}$ Fanni à qui son père vante les avantages d'une vie dictée par la raison: "Ah, mon père, les mouvements du coeur ne sont-ils pas préférables à ceux de l'esprit?". ${ }^{56}$ Il ne faut pas croire qu'a cet égard le théâtre se trouve totalement coupé du roman sadien. Le primat de la sensibilité se trouve en effet paradoxalement défendu par certains libertins. Ainsi dans La Philosophie dans le boudoir, le chevalier, qui partage certainement les théories anti-sociales des autres personnages apporte toutefois une réserve: “Laissons là les principes religieux, j'y consens mais n'abandonnons pas les vertus que la sensibilité nous inspire". ${ }^{57} \mathrm{Il}$ se peut que là se trouve la clef de l'apparente contradiction entre l'univers de ses romans et celui de son théâtre: Dans les drames de Sade comme dans ses romans le coeur de l'homme ne peut être considéré qu'en symbiose avec la nature dans une relation à la fois exaltante et terrible.

Car par-delà les influences rousseauistes et richardsoniennes, se perçoit en effet dans le théâtre du marquis de Sade la pensée philosophique qu'il exprime dans ses romans. Ces derniers vulgarisent les théories de la fin du XVIIIe siècle et particulièrement celles de d'Holbach, de Buffon et de Maupertuis. ${ }^{58}$

Rien de tout cela à première vue dans un théâtre désireux de respecter le code moralisateur du temps. Les invocations à l'Etre suprême -- concession au jargon révolutionnaire -- se font même entendre de temps en temps, fort discrètement il est vrai. ${ }^{59}$ Mais la Nature, au centre des romans de Sade, l'est aussi de son théâtre. C'est elle la force toute puissante de l'univers; c'est elle qui détermine les personnages de la dramaturgie sadienne: "Ce que je sais, ce que je sens, c'est qu'elle est la seule lumière qui nous soit donnée dans ce chaos d'évènements, où la nature nous jette" remarque un des personnages..$^{60}$ L'homme, jeté par accident dans l'univers n'a ni place privilégiée ni fin particulière. L'épicurisme dont la pensée de Sade est nourrie et qui se prête si bien dans ses romans à cette genèse matérialiste, colore aussi son théâtre:

Contemplez la nature

Offre-t-elle à vos yeux sous une règle sûre,

le fatigant tableau de l'uniformité

tout en elle est changeant, tout est variété

remarque Fonrose dans Le Capricieux.${ }^{61}$ Les conventions sociales, fruits de l'imagination de l'homme ne peuvent étouffer "la voix puissante" 
de la nature. Il est intéressant de noter que les deux pièces où se perçoit le mieux la philosophie matérialiste de Sade sont celles qui peuvent être considérées comme les plus romantiques; celles où il insiste particulièrement sur l'extrême sensibilité des héros; Henriette et SaintClair et Sophie et Desfrancs, ou Le Misanthrope par amour. Les deux pièces sont d'ailleurs très semblables. Elles tournent toutes deux autour du tabou de l'inceste.

Dans Henriette et Saint-Clair, les deux jeunes gens tombent amoureux et essaient de justifier leur amour même après avoir découvert qu'ils sont frère et soeur:

O nature - interroge Henriette - si je t'outrageais en aimant Saint-Clair, pourquoi me le laisserais-tu donc aimer sans remords? ... Et si je peux l'aimer encore sans t'outrager, quel est donc ce devoir cruel que les hommes osent dire être émané de toi? ${ }^{62}$

Et Saint-Clair à sa tante qui lui affirmait qu'un tel amour était contre nature:

Qui? moi, Madame? offenser la nature ... et qui m'a donc donné ce sentiment qui me dévore si ce n'est elle? De qui tiens-je ces mouvements impétueux qui m'entraînent malgré moi vers cet objet chéri dont je n'abandonnerai l'idée qu'à la mort? Ah, si j'outrageais la nature, ce coeur, ouvrage de ses mains, serait-il enflammé de cet amour que jamais rien ne vaincra dans lui ... En vain vous m'alléguerez les sophismes dont on combat les penchants de la nature. ${ }^{63}$

Dans le Misanthrope par amour la passion qui lie Sophie et Desfrancs ne s'éteint pas quand ils se découvrent père et fille. Toute puissante, la nature est amorale, indifférente aux conventions humaines. C'est elle, "cette voix qui parle au fond de nos coeurs ... cet organe dont le murmure est infaillible et qui jamais ne nous fait connaître de repentir" s'exclame un des personnages. ${ }^{64}$ Sade, il est vrai, finit par se souvenir du but édifiant de son théâtre et à la dernière scène du dernier acte des deux pièces, se produit le coup de théâtre par lequel SaintClair et Henriette apprennent qu'ils ne sont pas vraiment frère et soeur; Sophie et Desfrancs qu'ils ne sont pas vraiment père et fille. Mais jusqu'à ce que se produise ce miracle final, les quatre personnages ne vivent que par leur amour incestueux. Loin en effet de maudire les dieux ou la destinée, loin de se repentir ou d'être honteux d'un tel amour, les héros sadiens voient dans leur passion un signe de supériorité qui les élève au-dessus du commun et des préjugés sociaux. 
Le discours libertin est donc clairement perceptible chez les héros sensibles de ce théâtre. Quant aux libertins de moeurs qui appuient leur philosophie de leur conduite, ils n'en sont pas absents non plus. "L'homme est né pour jouir" affirme Fonrose ${ }^{65}$ et Oxtiern: "Mon bonheur, ma satisfaction voilà le but" ${ }^{\prime 66} \mathrm{Ce}$ dernier personnage rappelle d'ailleurs beaucoup les grands libertins des romans sadiens. Ainsi, par delà tout l'appareil néo-classique de ce théâtre et par delà la tentation du romantisme, se perçoivent les mêmes problèmes, les mêmes obsessions qui hantent les romans de Sade.

Et dans les rapports qui lient le théâtre aux romans de cet auteur, je tiens à souligner la part qu'il fait aux femmes. ${ }^{67}$

Si la dénonciation qu'il fait à maintes reprises du mariage forcé ${ }^{68}$ et de l'éducation au couvent ${ }^{69}$ est loin d'être nouvelle, il va beaucoup plus loin que ses contemporains dans sa critique de la condition sociale de la femme. Héritières de la tradition chrétienne, l'optique néo-classique et la philsophie de Rousseau, par leur culte de la famille et la déification du rôle de mère, incarcéraient la femme dans son rôle traditionnel. L'on sait le succès de ces idées sous Robespierre puis sous Napoléon. La science elle-même n'y échappa pas. Michel Foucault souligne dans L'Histoire de la folie que les manuels psychiatriques de l'époque avertissaient les femmes du danger du célibat et une enquête menée entre 1803 et 1804 dans les hôpitaux parisiens prouvait que le nombre des femmes célibataires aliénées était sept fois plus grand que celui des femmes mariées. ${ }^{70}$ Condorcet et Sade sont les seuls auteurs masculins de la période révolutionnaire à avoir violemment dénoncé la cruauté d'une société qui maintenait les femmes dans une totale dépendance. Si les longues tirades de Sade à ce sujet dans sa tragédie Jeanne Laisné et dans sa comédie Les Jumelles, nuisent idéniablement à l'effet dramatique, elles assurent à ces pièces leur seule originalité et méritent à leur auteur de figurer aux côtés de penseurs libéraux qui ont dénoncé l'oppression de la femme.

Dans Les Jumelles il brosse avec la plus grande sympathie le tableau de la femme assujettie à sa famille dans son enfance, à son mari dans sa vie adulte et enfin à ses enfants. Cette totale dépendance ne lui permet jamais d'éprouver le bonheur. La solitude des femmes, leur condition pitoyable, leur vieillesse sont évoquées avec compassion dans plusieurs de ses pièces. Les héroines du théâtre de Sade apportent d'autre part un souffle d'air frais dans la litérature néo-classique. L'auteur ne semble en effet guère satisfait de l'identification que fait la société patriarcale de la masculinité avec la compétence et le goût du risque et de la féminité avec l'incompétence, la passivité, la modestie. Il dote ses héroines de capacités rarement associées à leur sexe. La 
baronne de Florange dans Le Capricieux décide de séduire le libertin Fonrose pour qu'il ne fasse pas le malheur de la jeune Céline qui en est aveuglément amoureuse. Dans Le Mariage du siècle Destournelle empoisonne la femme de l'homme qu'elle aime et le conduit à la folie. Elle s'apparente aux grandes héroines des romans sadiens, à celles qui, comme Juliette ou Clairvill, ayant compris les ressorts d'une société corrompue, vont surmonter l'handicap de leur sexe pour jouer le jeu social mieux que les hommes. Dans Le Comte Oxtiern, Ernestine séduite et enlevée par Oxtiern refuse le remède traditionnel, c'est-à-dire le mariage: Non, non Oxtiern, ce n'est pas ta main que je veux, c'est ta mort. ${ }^{71}$ Et à Oxtiern qui lui faisait remarquer que son destin ne dépendait que de lui:

Cette femme ne dépend que d'elle. Elle ne se doit qu'à elle: elle seule règlera son sort. ${ }^{72}$

Et refusant d'épouser son séducteur, elle décline également l'aide que veut lui apporter son père et prend sa vengeance entre ses propres mains. Elle provoque Oxtiern en duel.

Dans Euphémie de Melun, Euphémie pour se rendre digne de celui qu'elle aime apprend non par l'art du ménage mais celui du combat et c'est en armure et les armes à la main qu'elle est faite prisonnière.

Le sujet de la résistance de Jeanne Hachette au siège de Beauvais se prêtait particulièrement bien aux idées de l'auteur. Jeanne, refusant le rôle traditionnel auquel veut la confiner son père, va organiser la défense de la ville:

Ce sexe quoique faible a reçu la valeur,

L'ambition, l'orgueil, le zèle, le courage,

L'amour de son pays comme l'autre en partage ${ }^{73}$

Elle incite ses compagnes à la résistance, dissipe leurs hésitations. Ainsi à une amie qui demandait:

Notre sexe a-t-il des droits à la victoire? ${ }^{74}$

Jeanne de répondre:

Crois qu'il en a toujours aux palmes de la gloire;

Crois qu'il n'est grand qu'alors qu'il a su s'élever

Au-dessus de lui-même, et qu'il a pu prouver

A ce rival si vain qui l'abaisse et l'outrage, 
En doutant sans raison de son noble courage, A ce sexe orgueilleux qui

l'enchaîne aujourd'hui,

Qu'il a, quand il le faut, des vertus comme lui ${ }^{75}$

A Emilie qui lui objectait son amour:

Ah! parle moins d'amour et sois plus citoyenne ${ }^{76}$

Car le malheur public fournit enfin l'occasion aux femmes de sortir de leur rôle traditionnel.

$C^{\prime}$ est au sein du malheur qu'il faut briser ses chaînes ${ }^{77}$ encourage Jeanne. Et l'on voit Jeanne Laisné s'élancer

sur les remparts, à la tête des femmes, au milieu du fracas le plus terrible de l'artillerie et d'une nouvelle nuée de bombes dont quelques unes éclatent sur le bataillon. Plusieurs femmes sont renversées; les autres leur passent sur le corps, et rien n'arrête l'élan de leur marche. ${ }^{78}$

Est-ce une coincidence si cette pièce fut écrite entre 1789 et 1791 ? ou peut-on y voir l'hommage que Sade rendit à la participation active des femmes à la Révolution? Cette pièce devrait alors être considérée comme une pièce politique, document historique qui pourrait s'ajouter à ceux accumulés par les historiens qui, depuis une quinzaine d'années, se penchent sur cette contribution des femmes à la Révolution. Peuton alors s'étonner que cette pièce si chère à son auteur fut dédaignée par la période révolutionnaire et par l'Empire?

Dans la part qu'il faisait aux femmes, tout comme dans ses conceptions de la nature, Sade allait en effet à contre courant de son temps. Son théâtre qui se voulait pourtant oeuvre de conciliation ne fait que confirmer l'écart qui régnait entre lui et ses contemporains. On ne peut donc s'empêcher de se demander si, quand il offrait successivement son théâtre à la Révolution et à l'Empire il se rendait compte de l'étrangeté d'un tel don?

MARIE-FRANCE SILVER

Université York 


\section{NOTES}

1. D.F. Sade Théâtre (dans:) D.F. Sade Oeuvres complètes, Paris, Pauvert. vol. 32, pp. 129-130.

2. G. Lely, La Vie du marquis de Sade, Paris, 1976, Cercle du livre précieux, vol. II, p. 204.

3. La comédie-féerie: Azélis ou la coquette punie fut acceptée par le théâtre comique et lyrique de la rue de Bondi. Le Misanthrope par amour fut reçu par la Comédie Française.

4. Théâtre, vol. 32, p. 132.

5. F. Laugaa-Trout, Lectures de Sade, Armand Colin, 1973, p. 51.

6. Théâtre, vol. 32, p. 125.

7. Laugaa-Trout, op. cit., pp. 51-53.

8. Ibidem, pp. 53-54.

9. Jean Tulard, "Sade et la censure sous le premier empire" (dans:) Le Marquis de Sade, Colloque d'Aix, A. Colin, 1968.

10. Lely, op. cit., vol. II, pp. 537-538.

11. L'expression est de Michel Vovelle dans Théodore Desorgues ou la désorganisation, Le Seuil, p. 198.

12. Ibidem, pp. 200-201.

13. Tancrède, (dans:) Théâtre, Vol. 33, p. 198. Aussi L'Union des arts. Ibidem, Vol, 34, pp. 312-314.

14. Jean Tulard, op. cit., p. 215.

15. Lely, op. cit., vol. II, pp. 577, 579.

16. Ibidem, vol. II, p. 204. Aussi Théâtre, vol. 33, p. 170.

17. Jean Tulard, op. cit., pp. 216-217.

18. Théâtre, vol. 32, p. 18.

19. Lely, op. cit., vol. II, p. 558.

20. Ibidem, op. cit., vol. II, p. 569. Aussi Marquis de Sade, Journal Inedit, Gallimard, 1970, pp. 140-141-142.

21. La fète de l'amitié dans Théatre, vol. 35, p. 434.

22. Lely, op. cit., vol. II, p. 581.

23. F. Dornic, La France de la Révolution, Culture, Art, Loisirs, Paris, 1970, p. 224.

24. Le Boudoir, (dans:) Théâtre, vol. 33, p. 90.

25. Ibidem, p. 91

26. Théâtre, vol. 35, pp. 262- 263.

27. Ibidem, vol. 34, p. 333.

28. Ibidem, vol. 35 , p. 10. 
29. Ibidem, vol. 33, p. 264.

30. Ibidem, vol. 33, p. 13.

31. Ibidem, vol. 34 , p. 134.

32. Ibidem, vol. 34 , p. 106.

33. Ibidem, vol. 34, p. 107.

34. Le Comte Oxtiern, Acte I, scène V.

35. Le Prévaricateur, Acte V, scène IV. Aussi Henriette et Saint-Clair

36. Le Comte Oxtiern. Aussi Le Misanthrope par amour, Acte I, scène IV.

37. Jean Fabre, "Sade et le roman noir", (dans:) Le Marquis de Sade, Colloque d'Aix, A. Colin, Paris, 1968, pp. 260-261.

38. L'Eqarement de l'infortune.

39. Fanni ou les effets du desespoir.

40. Henriette et Saint-Clair.

41. Le Comte Oxtiern.

42. Jeanne Laisné.

43. L'Eqarement de l'infortune.

44. La Tour enchantée.

45. Le Mariage du siècle.

46. Fanni ... Aussi Henriette et Saint-Clair.

47. Consultez l'essai Sade, dramaturge de ses Carceri, (dans:) B. Didier, Sade, Paris, Deuvil, 1976.

48. La Fète de l'amitié.

49. Fanni ...

50. L 'Eqarement.

51. Ibidem, Acte I, scène I.

52. Ibidem, Acte II, scène I.

53. Henriette et Saint-Clair, Acte IV, scène II.

54. Ibidem, Acte I, scène IV.

55. Ibidem.

56. Fanni ... Acte I, scène V.

57. La Philosophie dans le boudoir, III dialogue.

58. Voir J. Deprun, "Sade et la philosophie biologique de son temps", (dans:) Le Marquis de Sade, Colloque d'Aix, op. cit. Aussi M.F. Silver, "Sade et les théories scientifiques de son temps", (dans:) Actes du Colloque de la Société d'Etudes méditeranéennes, Toronto, 1983.

59. Henriette et Saint-Clair, Acte III, scène V; Acte IV, scène II.

60. Ibidem, Acte III, scène II. 
61. Le Capricieux, Acte IV, scène III.

62. Henriette et Saint-Clair, Acte II, scène III.

63. Ibidem, Acte III, scène II.

64. Ibidem, Acte II, scène IV.

65. Le Capricieux, Acte IV, scène V.

66. Le Comte Oxtiern, Acte II, scène I.

67. M.F. Silver, "Les héroines des romans sadiens", communication présentée au congrès annuel des Etudes canadiennes du XVIIIe siècle, Winnipeg, 1984.

68. Les Jumelles, Acte I, scène III. Aussi Fanni ...

69. Henriette et Saint-Clair, Acte II, scène II.

70. Michel foucauld, Histoire de la folie à l'âge classique, p. 268, Union Générale d'Editions, 1961.

71. Le Comte Oxtiern, Acte II, scène VIII.

72. Ibidem. Acte II, scène V.

73. Jeanne Laisné, Acte IV, scène III.

74. Ibidem, Acte II, scène I.

75. Ibidem.

76. Ibidem, Acte II, scène I.

76. Ibidem, Acte II, scène I.

77. Ibidem.

78. Jeanne Laisné, Acte IV, scène VI. 